\title{
Membrane Flexibility and Exercise: A Guide to Type 2 Diabetes Mellitus
}

\section{Rob Weijers*}

Teaching Hospital, Onze Lieve Vrouwe Gasthuis, Amsterdam, The Netherlands

\begin{abstract}
During the last two decades the discipline of biology developed the pacemaker hypothesis, which notes that the molecular composition of the mammalian lipid bilayer is centrally involved in regulating the metabolic activity of cells. At the same time, the discipline of physical chemistry demonstrated that the ratio of unsaturated to saturated fatty acyl chains regulates the mutual distance of these chains in a bilayer membrane, and consequently affects the flexibility of the membrane. Based on these innovative results, an assessment of the deformability of the red blood cell provides reliable information about glucose disposal during the treatment of type 2 diabetes mellitus, as well as during aerobic exercise.
\end{abstract}

Keywords: Cell membrane flexibility; Erythrocyte deformability; Exercise; Glucose transporter; Irisin; Pacemaker hypothesis; Phospholipids; Type 2 diabetes mellitus; Unsaturated fatty acid

\section{Introduction}

The most important goal of a science magazine is probably to provide its readers with valuable insights into the coherence of the natural sciences. This also applies to the journals publishing research in the landscape of diabetes mellitus type 2. Given this viewpoint, the aim of this contribution is to shed light on two innovative scientific findings: (1) the observation of a consistent pattern of acyl composition of membrane bilayers among different-sized mammals that modulate their basal metabolic rate, and (2) the observation of a positive correlation between the surface of the cross-section of the hydrocarbon portion of a phospholipid molecule and its degree of unsaturation. Combining this new information leads to a major advance in understanding how exercise favors the well being of patients with type 2 diabetes mellitus.

\section{Membrane Pacemaker Theory of Metabolism}

The first area of pioneering research regards the application of allometry, which describes changes in the proportions of various parts of an organism as a consequence of growth. Both an allometric comparison of metabolism in a wide variety of species ranging in weight from $\sim 1$ to $10^{5} \mathrm{~g}$ body mass encompassing an evolutionary period of $\sim 150$ million years and comparison of ectotherms with endotherms led to the hypothesis that the amount of membranes and their acyl composition, and particularly the relative balance between monounsaturated and polyunsaturated acyl chains, are a pacemaker for metabolic activity [1]. Evidence supporting the membrane pacemaker theory is generally based on the observation that although there is essentially no difference in the percentage of unsaturated fatty acids in cellular membranes of species with dramatically different basal metabolic rates, it is consistently observed that species with a high basal metabolic rate have a significantly greater density of carboncarbon double bonds, and especially docosahexaenoic acid, in their cellular membrane bilayers than species with a lower basal metabolic rate. Therefore, we must replace the view that membrane bilayers are relatively passive components of eukaryotic cells with the concept that the relative balance between monounsaturated and long-chain polyunsaturated acyl chains in membrane bilayers is a fundamental determinant of the metabolic rate of a species $[2,3]$.

\section{Membrane Flexibility}

The second area of innovative research relates to the scientific understanding of the molecular nature of a glycerophospholipid bilayer membrane. The two fatty acyl chains of a phospholipid yield, in essence, a roughly cylindrical molecule (the hydrocarbon region) that can easily pack in parallel to form extended sheets of membranes due to hydrogen bonds. The interaction energy (U) between two carbon atoms of these nearby chains is the sum of the repulsive force and the attractive force, and is described mathematically as

$$
\mathrm{U}=11.5 \times 10^{-6} / \mathrm{r}^{12}-5.96 \times 10^{-3} / \mathrm{r}^{6} \mathrm{~kJ} / \mathrm{mol}
$$

where, $\mathrm{r}$ is the distance in nanometers between the centers of the two carbon atoms [4]. To what extent calculations based on artificial models do properly describe intermolecular interactions, especially the London-van der Waals forces, was recently presented by atomic force microscopy measurement data of intermolecular interactions between two $\mathrm{CO}$ molecules which indicated the correctness of the LennardJones potential [5].

The most basic structural result obtained by X-ray scattering from oriented bilayers in model membrane systems is a particularly central quantity: the area (A) per lipid molecule (i.e. the surface of the cross-section of the hydrocarbon region part of the phospholipid molecule) [6,7] (Figure 1). Recent studies have highlighted the physical property that unsaturated hydrocarbon tails clearly lead to a greater value for A compared with saturated tails, as depicted in table 1. Thus, the replacement of saturated fatty acyl chains with unsaturated acyl chains results in a decrease in interaction energy per pair of fatty acyl chains, and consequently to increased membrane flexibility. A unique characteristic of the bilayer membrane design is the reversibility of its flexibility. An increase in the unsaturation index (the number of carbon-carbon double bonds per 100 acyl chains) creates an increase in

*Corresponding author: Rob Weijers, Onze Lieve Vrouwe Gasthuis, Oosterparkstraat 9, PO Box Box 95500, 1090 HM Amsterdam, The Netherlands, Tel: +31-30-6362637; E-mail: robw01@xs4all.nl

Received January 03, 2013; Accepted February 18, 2013; Published February 23, 2013

Citation: Weijers R (2013) Membrane Flexibility and Exercise: A Guide to Type 2 Diabetes Mellitus. J Diabetes Metab S10: 003. doi:10.4172/2155-6156.S10-003

Copyright: ( 2013 Weijers R. This is an open-access article distributed under the terms of the Creative Commons Attribution License, which permits unrestricted use, distribution, and reproduction in any medium, provided the original author and source are credited. 


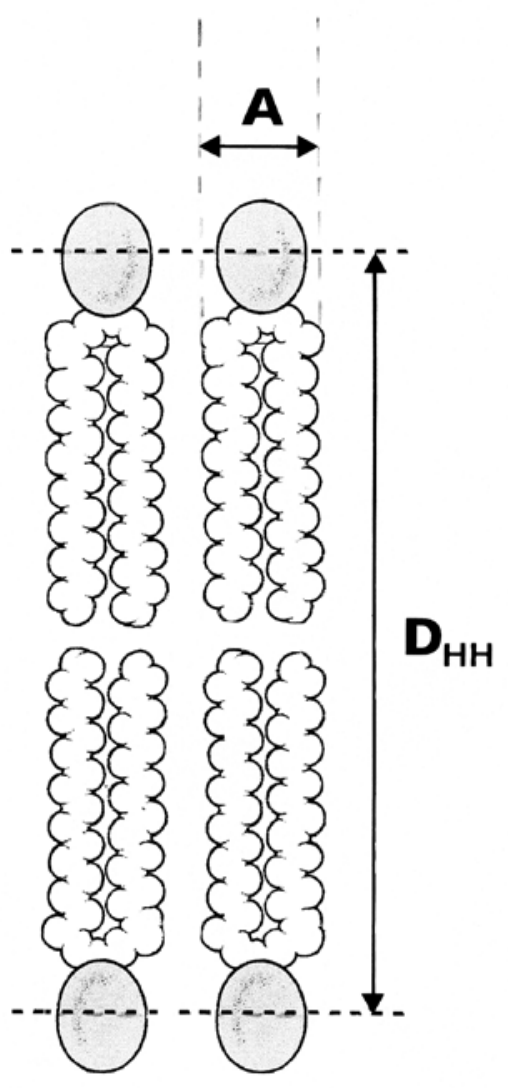

Figure 1: Schematic representation of part of a lipid bilayer, including the interfacial area (A) per lipid molecule (i.e. the surface of the cross-section of the cylindrical hydrocarbon part of the phospholipid molecule) and the head-tohead distance $\left(D_{H H}\right)$ across the lipid bilayer.

\begin{tabular}{|l|c|c|}
\hline Lipid & DMPC & DOPC \\
\hline References & 4 & 5 \\
\hline Fatty acid structure & C14:0 & C18:1 \\
\hline Area per lipid molecule $(\mathrm{A})\left(\AA^{2}\right)$ & 60.6 & 72.5 \\
\hline Inter C-C distance $(\AA)^{\star}$ & 4.39 & 4.80 \\
\hline Interaction energy U $(\mathrm{kJ} / \mathrm{mol})^{\dagger}$ & -0.61 & -0.41 \\
\hline Decrease in interaction energy $(\mathrm{kJ} / \mathrm{mol})$ & & $32.8 \%$ \\
\hline
\end{tabular}

*The calculation of the inter $\mathrm{C}-\mathrm{C}$ distance is from the area per lipid molecule $(\mathrm{A})\left(\AA^{2}\right)$ †The interaction energy is given by: $U=11.5 \times 10^{-6} / \mathrm{r}^{12}-5.96 \times 10^{-3} / \mathrm{r}^{6} \mathrm{~kJ} / \mathrm{mol}$ DMPC: Dimyristoyl-Phosphatidylcholine; DOPC: Dioleoyl-Phosphatidylcholine

Table 1: Experimental data of fully hydrated fluid phase, artificial phosphatidylcholine lipid bilayers.

membrane flexibility, and a decrease in the unsaturation index results in a decrease in membrane flexibility.

Within the framework of the previous sections, the conclusion is that a cause-effect relationship exists between a cell's membrane flexibility and the pacing of its metabolism. An important issue of this characteristic involves the role of highly unsaturated fatty acids (generally, docosahexaenoic acid [C22:6n-3]) in biological membranes. That role may be to support the function of certain membrane proteins that require large cross-sectional lipid areas [8,9].

\section{Glucose Transport Across Cell Membranes}

Specific transporter proteins such as Solute carrier family 2, facilitated glucose transporter members (GLUTs) are required for facilitated glucose diffusion into cells [10]. Facilitated diffusion rates display saturation behavior similar to that observed in the binding of substrate by enzymes. Because the Michaelis-Menten constants for all glucose transporters except GLUT2 are below the normal range of blood glucose concentrations, glucose transporters function at rates close to maximal velocity and their degree of cell surface expression greatly influences the rate of glucose uptake into cells [11]. Glucose transporters are integral membrane proteins and, based on a GLUT1 cross-sectional area of approximately $1,100 \AA$ [12], one glucose transporter molecule covers an area of approximately 17 molecules of a phosphatidylcholine bilayer with saturated fatty acyl chains. Therefore, the process of glucose transporter insertion into a bilayer membrane requires that the membrane is highly flexible.

GLUT4 differs from other glucose transporters in that approximately $90 \%$ of it is sequestered in intracellular vesicles in the absence of insulin. On stimulation by insulin, the intracellular vesicles are transported to the inner leaf of muscle plasma membranes, culminating finally in membrane fusion [13]. As described by the 'stalk-pore' hypothesis, this fusion process also requires a highly flexible bilayer membrane [14].

Based on the presented discoveries, we suggest that a shift from unsaturation toward saturation in phospholipid membranes counteracts both the pore formation of a muscle plasma membrane fused with an intracellular GLUT4-containing vesicle, and the machinery responsible for insulin-independent glucose transporter insertion into a plasma membrane. This phenomenon leads to the proposal that the phospholipid acyl composition of the cell membrane is an important determinant of its metabolic activity because this composition regulates the number of glucose transporters per unit of cell surface area (i.e. the glucose transport capacity). Under normal cellular conditions, the eukaryotic cell extracts 32 ATP molecules (the final fuel of metabolic activity) through the combination of glycolysis, the tricarboxylic acid cycle, electron transport, and oxidative phosphorylation from each transported glucose molecule.

Regarding the aetiology of type 2 diabetes mellitus, we recapitulate that already in the pre-diabetic phase, reductions in basal rates of mitochondrial ATP synthesis in skeletal muscle caused by decreased mitochondrial activity [15-17] serve to drive hepatic lipogenesis. This process leads to the gradual elevation of plasma free fatty acids $[18,19]$ to yield more energy in the form of ATP, which develops a shift of membrane phospholipids from unsaturated fatty acyl chains toward saturated fatty acyl chains, resulting in decreased flexibility and consequently in a decreased flux of glucose into the cells. What is clinically exciting is that the regulation of membrane flexibility may reverse the incidence of type 2 diabetes in high-risk individuals.

\section{Membrane Flexibility and Exercise}

Although the role of irisin in the conversion of white adipose tissue into brown adipose tissue is still being debated [20], acute exercise training showed direct effects on 'browning' of white fat [21]. Hamilton et al. [22] were able to quantify an average number of carbon-carbon double bonds from magnetic resonance spectra of human tissue and reported that brown adipose tissue was more saturated than white adipose tissue, an observation consistent with previous findings [23] This process induces a shift of membrane phospholipids from saturation toward unsaturation and promotes more membrane flexibility.

The element of choice for the assessment of membrane flexibility is the red blood cell bilayer membrane. Nishida et al. showed that 
performing aerobic exercise is a unique approach that may help to increase insulin-dependent and insulin-independent glucose disposal in healthy humans [24]. Moreover, three observational studies about the long term effects of diet and exercise in individuals at high risk for type 2 diabetes demonstrated that lifestyle changes reduce the incidence of this disease [25-27]. However, the aforementioned studies did not study the effect of aerobic exercise on membrane deformability. Cho et al. studied hemorrheological disorders in diabetes mellitus [28]. The data reviewed herein demonstrated that reduced red cell deformability was associated with retinal failure in diabetic retinopathy and renal failure in diabetic nephropathy. However, those studies did not investigate the impact of exercise on the deformability of the red blood cell. Finally, they concluded that abnormal glycation stiffens the erythrocyte membrane. This conclusion, however, is based on a fundamental flaw because the electrostatic interactions within a folded protein structure contribute $\sim 20 \mathrm{~kJ} / \mathrm{mol}$ of stabilization energy, while the van der Waals interactions between fatty acyl chains individually contribute $0.4-4.0 \mathrm{~kJ} / \mathrm{mol}$ of stabilization energy [4]. Since the driving force of membrane flexibility is formed by the biomolecules with the lowest stabilization energy, the deformability of bilayer membranes depends solely on the lipid composition of their hydrocarbon tails. This misinterpretation may be appropriate cause to disavow the assessment of erythrocyte deformability. I propose to use this parameter to monitor red blood cell flexibility during the treatment of patients with type 2 diabetes mellitus, and to obtain a better understanding of how aerobic exercise improves glucose disposal in the human body.

The role of cholesterol in bilayer membranes has been extensively investigated for many years. Now, new techniques are deepening our insight into the dynamics of membrane organization. In the present model, membranes are occupied by fluctuating nanoscale assemblies of sphingolipids, cholesterol and proteins that can be stabilized into platforms that are important in signaling, viral infection and membrane trafficking [29]. Moreover, new data indicate that the assumption that cholesterol is a principal modulator of bilayer thickness is not valid and suggest that the cholesterol content in cell membranes is not modulated for the purpose of controlling bilayer thickness [30].

\section{Conclusions}

New experimental data obtained outside the traditional research field of type 2 diabetes resulted in the insight that biological bilayer membranes serve not only to package the content of living cells, but also to regulate their metabolic activities by changing the unsaturation index of lipid bilayers. Thus, assessment of red blood cell deformability may constitute a novel approach to offer guidance to physicians for the treatment of patients with type 2 diabetes mellitus and to monitor the effects of a lifestyle intervention (i.e. weight reduction induced by aerobic exercise) in overweight patients.

\section{References}

1. Hulbert AJ, Else PL (1999) Membranes as possible pacemakers of metabolism J Theor Biol 199: 257-274.

2. Hulbert AJ (2003) Life, death and membrane bilayers. J Exp Biol 206: 23032311.

3. Hulbert AJ (2007) Membrane fatty acids as pacemakers of animal metabolism Lipids 42: 811-819.

4. Garrett RH, Grisham CM (1999) Biochemistry (2ndedn), Saunders College Publishing: Fort Worth: 2-33.

5. Sun Z, Boneschanscher MP, Swart I, Vanmaekelbergh D, Liljeroth P (2011) Quantitative atomic force microscopy with carbon monoxide terminated tips. Phys Rev Lett 106: 046104
6. Kucerka N, Liu Y, Chu N, Petrache HI, Tristram-Nagle S, et al. (2005) Structure of fully hydrated fluid phase DMPC and DLPC lipid bilayers using X-ray scattering from oriented multilamellar arrays and from unilamellar vesicles. Biophys J 88: 2626-2637.

7. Nagle JF, Tristram-Nagle S (2000) Structure of lipid bilayers. Biochim Biophys Acta 1469: 159-195.

8. Rajamoorthi K, Petrache HI, Mclntosh TJ, Brown MF (2005) Packing and viscoelasticity of polyunsaturated omega- 3 and omega- 6 lipid bilayers as seen by (2)H NMR and X-ray diffraction. J Am Chem Soc 127: 1576-1588.

9. Weijers RN (2012) Lipid composition of cell membranes and its relevance in type 2 diabetes mellitus. Curr Diabetes Rev 8: 390-400.

10. Garrett RH, Grisham CM (1999) Biochemistry. (2ndedn), Saunders College Publishing: Fort Worth: 296-326.

11. Bouché C, Serdy S, Kahn CR, Goldfine AB (2004) The cellular fate of glucose and its relevance in type 2 diabetes. Endocr Rev 25: 807-830.

12. Salas-Burgos A, Iserovich P, Zuniga F, Vera JC, Fischbarg J (2004) Predicting the three-dimensional structure of the human facilitative glucose transporter glut1 by a novel evolutionary homology strategy: insights on the molecular mechanism of substrate migration, and binding sites for glucose and inhibitory molecules. Biophys J 87: 2990-2999.

13. Shepherd PR, Kahn BB (1999) Glucose transporters and insulin actionimplications for insulin resistance and diabetes mellitus. N Engl J Med 341 248-257.

14. Marrink SJ, Mark AE (2003) The mechanism of vesicle fusion as revealed by molecular dynamics simulations. J Am Chem Soc 125: 11144-11145.

15. Kelley DE, He J, Menshikova EV, Ritov VB (2002) Dysfunction of mitochondria in human skeletal muscle in type 2 diabetes. Diabetes 51: 2944-2950.

16. Petersen KF, Dufour S, Befroy D, Garcia R, Shulman GI (2004) Impaired mitochondrial activity in the insulin-resistant offspring of patients with type 2 diabetes. N Engl J Med 350: 664-671.

17. Petersen KF, Dufour S, Shulman GI (2005) Decreased insulin-stimulated ATP synthesis and phosphate transport in muscle of insulin-resistant offspring of type 2 diabetic parents. PLoS Med 2: e233.

18. Lohninger A, Radler $U$, Jinniate S, Lohninger S, Karlic H, et al. (2009) Relationship between carnitine, fatty acids and insulin resistance. Gynäkol Geburtshilfliche Rundsch 49: 203-205.

19. Boden G, Shulman GI (2002) Free fatty acids in obesity and type 2 diabetes: defining their role in the development of insulin resistance and beta-cel dysfunction. Eur J Clin Invest 32: 14-23.

20. Timmons JA, Baar K, Davidsen PK, Atherton PJ (2012) Is irisin a human exercise gene? Nature 488: E9-10.

21. Huh JY, Panagiotou G, Mougios V, Brinkoetter M, Vamvini MT, et al. (2012) FNDC5 and irisin in humans: I. Predictors of circulating concentrations in serum and plasma and II. mRNA expression and circulating concentrations in response to weight loss and exercise. Metabolism 61: 1725-1738.

22. Hamilton G, Smith DL Jr, Bydder M, Nayak KS, Hu HH (2011) MR properties of brown and white adipose tissues. J Magn Reson Imaging 34: 468-473.

23. Zingaretti MC, Crosta F, Vitali A, Guerrieri M, Frontini A, et al. (2009) The presence of UCP1 demonstrates that metabolically active adipose tissue in the neck of adult humans truly represents brown adipose tissue. FASEB $\mathrm{J} 23$ 3113-3120.

24. Nishida Y, Tokuyama K, Nagasaka S, Higaki Y, Shirai Y, et al. (2004) Effect of moderate exercise training on peripheral glucose effectiveness, insulin sensitivity, and endogenous glucose production in healthy humans estimated by a two-compartment-labeled minimal model. Diabetes 53: 315-320.

25. Pan XR, Li GW, Hu YH, Wang JX, Yang WY, et al. (1997) Effects of diet and exercise in preventing NIDDM in people with impaired glucose tolerance. The Da Qing IGT and Diabetes Study. Diabetes Care 20: 537-544.

26. Tuomilehto J, Lindström J, Eriksson JG, Valle TT, Hämäläinen H, et al. (2001) Prevention of type 2 diabetes mellitus by changes in lifestyle among subjects with impaired glucose tolerance. N Engl J Med 344: 1343-1350.

27. Knowler WC, Barrett-Connor E, Fowler SE, Hamman RF, Lachin JM, et al (2002) Reduction in the incidence of type 2 diabetes with lifestyle intervention or metformin. N Engl J Med 346: 393-403. 
Citation: Weijers R (2013) Membrane Flexibility and Exercise: A Guide to Type 2 Diabetes Mellitus. J Diabetes Metab S10: 003. doi:10.4172/21556156.S10-003

Page 4 of 4

28. Cho YI, Mooney MP, Cho DJ (2008) Hemorheological disorders in diabetes mellitus. J Diabetes Sci Technol 2: 1130-1138.

29. Simons K, Gerl MJ (2010) Revitalizing membrane rafts: new tools and insights. Nat Rev Mol Cell Biol 11: 688-699.
30. Mitra K, Ubarretxena-Belandia I, Taguchi T, Warren G, Engelman DM (2004) Modulation of the bilayer thickness of exocytic pathway membranes by membrane proteins rather than cholesterol. Proc Natl Acad Sci U S A 101 4083-4088. 